\title{
AN INVERSE SPECTRAL PROBLEM FOR DIFFERENTIAL OPERATORS WITH INTEGRAL DELAY
}

\author{
YU. KURYSHOVA
}

\begin{abstract}
The uniqueness theorem is proved for the solution of the inverse spectral problem for second-order integro-differential operators on a finite interval. These operators are perturbations of the Sturm-Liouville operator with convolution and one-dimensional operators. The main tool is an integral transform connected with solutions of integrodifferential operators.
\end{abstract}

\section{Introduction}

Consider the problem $L=L(q, M, R, V)$ of the form

$$
\begin{aligned}
\ell y(x) & :=-y^{\prime \prime}(x)+q(x) y(x)+\int_{0}^{x} M(x-t) y(t) d t+R(x) \int_{0}^{\pi} V(t) y(t) d t=\lambda y(x), 0 \leq x \leq \pi, \\
y(0) & =y^{\prime}(0)=0
\end{aligned}
$$

where $\lambda$ is the spectral parameter, and $q, M, R, V$ are continuous functions. The operator $\ell$ is a perturbation of the Sturm-Liouville operator with convolution and one-dimensional operators. We study the inverse spectral problem for $L$. Inverse problems of spectral analysis consist in recovering operators from their spectral characteristics. Such problems often appear in many branches of natural sciences and engineering. For the Sturm-Liouville differential operator, inverse spectral problems have been studied fairly completely (see the monographs [1]-[5] and the references therein). Inverse problems for integro-differential and integral operators are much more difficult for investigating, and nowadays there are only several isolated results related to these "non-local" inverse problems (see [6]-[9] and the references therein). The integro-differential operator $\ell$, considered in the present paper, is the one-dimensional perturbation of the Volterra integro-differential operator. We study the inverse problem of recovering the perturbation provided that the Volterra part is known a priori. In order to formulate the inverse problem for (1)-(2) we first introduce the spectral data for $L$.

2000 Mathematics Subject Classification. 47G20, 45J05, 44A15.

Key words and phrases. Integro-differential operators, integral transforms, inverse spectral problems, uniqueness theorem. 
Let $u(x, \lambda)$ be the solution of the Cauchy problem

$$
-u^{\prime \prime}(x, \lambda)+q(x) u(x, \lambda)+\int_{0}^{x} M(x-t) u(t, \lambda) d t+R(x)=\lambda u(x, \lambda), \quad u(0, \lambda)=u^{\prime}(0, \lambda)=0 .
$$

Denote

$$
\Delta(\lambda):=1-\int_{0}^{\pi} V(t) u(t, \lambda) d t .
$$

The function $\Delta(\lambda)$ is entire in $\lambda$, and its zeros $\left\{\lambda_{n}\right\}$ coincide with the eigenvalues of $L$. The function $\Delta(\lambda)$ is called the characteristic function of $L$.

We note that if $V(x) \equiv 0$ or/and $R(x) \equiv 0$, then $\Delta(\lambda) \equiv 1$ (if $R(x) \equiv 0$, then $u(x, \lambda) \equiv 0$, since the Cauchy problem is equivalent to the homogeneous integral Volterra equation of second kind), and $L$ has no eigenvalues. In order to avoid this trivial case we will assume in the sequel that

$$
R(x) \sim d_{0} x^{\alpha}, \quad V(\pi-x) \sim d_{1} x^{\beta}, x \rightarrow 0, d_{0} d_{1} \neq 0,
$$

where $\alpha, \beta \geq 0$. In this case $L$ has a countable set of eigenvalues $\left\{\lambda_{n}\right\}_{n \geq 1}$. Moreover, if $\kappa_{n} \geq 1$ is a multiplicity of the zero $\lambda_{n}$ of $\Delta(\lambda)$, then the functions

$$
u_{j n}(x):=\frac{\partial^{j} u(x, \lambda)}{\partial \lambda^{j}} \mid \lambda=\lambda_{n}, \quad j=\overline{0, \kappa_{n}-1}
$$

are root functions of $L$. Denote $\alpha_{j n}:=u_{j n}(\pi), n \geq 1, j=\overline{0, \kappa_{n}-1}$. The data $S:=\left\{\lambda_{n}, \alpha_{j n}\right\}_{n \geq 1, j=\overline{0, \kappa_{n}-1}}$ are called the spectral data of $L$.

The inverse problem is formulated as follows: Let $q$ and $M$ be known a priori and fixed. Given the spectral data $S$, construct $R$ and $V$.

The goal of the present paper is to prove the uniqueness theorem for the solution of this inverse problem. For this purpose together with $L$ we consider a problem $\tilde{L}:=L(q, M, \tilde{R}, \tilde{V})$. We agree that if a certain symbol $a$ denotes an object related to $L$, then $\tilde{a}$ will denote a similar object related to $\tilde{L}$. Now we formulate the main result of the paper.

Theorem 1. If $S=\tilde{S}$, then $R=\tilde{R}$ and $V=\tilde{V}$. Thus, the specification of the spectral data $S$ uniquely determines the functions $R$ and $V$.

In Section 2 we establish some auxiliary propositions, and in Section 3 we provide the proof of Theorem 1.

\section{Auxiliary propositions}

Denote

$$
\ell_{1} y(x):=-y^{\prime \prime}(x)+q(x) y(x)+\int_{0}^{x} M(x-t) y(t) d t
$$


Let $S(x, \lambda)$ be the solution of the Cauchy problem

$$
\ell_{1} S(x, \lambda)=\lambda S(x, \lambda), \quad S(0, \lambda)=0, S^{\prime}(0, \lambda)=1
$$

By the same arguments as in [7] and [9] one gets that the following representation holds

$$
S(x, \lambda)=\frac{\sin \rho x}{\rho}+\int_{0}^{x} K(x, t) \frac{\sin \rho t}{\rho} d t, \quad \lambda=\rho^{2},
$$

where $K(x, t)$ is a twice continuously differentiable function which does not depend on $\lambda$, and $K(x, 0)=0$.

Lemma 1. The following relations are valid

$$
\begin{gathered}
K_{t t}(x, t)-K_{x x}(x, t)+q(x) K(x, t)+M(x-t)+\int_{t}^{x} M(x-\xi) K(\xi, t) d \xi=0, \\
q(x)=2 \frac{d K(x, x)}{d x} .
\end{gathered}
$$

Proof. Differentiating (7) twice with respect to $x$, we get

$$
\begin{aligned}
& S^{\prime}(x, \lambda)=\cos \rho x+K(x, x) \frac{\sin \rho x}{\rho}+\int_{0}^{x} K_{x}(x, t) \frac{\sin \rho t}{\rho} d t \\
& S^{\prime \prime}(x, \lambda)=-\rho \sin \rho x+\frac{d}{d x}\left(K(x, x) \frac{\sin \rho x}{\rho}\right)+K_{x}(x, t)_{\mid t=x} \frac{\sin \rho x}{\rho}+\int_{0}^{x} K_{x x}(x, t) \frac{\sin \rho t}{\rho} d t .
\end{aligned}
$$

Substituting into (6) we calculate

$$
\begin{aligned}
\rho \sin \rho x & -\frac{d}{d x}\left(K(x, x) \frac{\sin \rho x}{\rho}\right)-K_{x}(x, t)_{\mid t=x} \frac{\sin \rho x}{\rho}-\int_{0}^{x} K_{x x}(x, t) \frac{\sin \rho t}{\rho} d t \\
+ & q(x) \frac{\sin \rho x}{\rho}+q(x) \int_{0}^{x} K(x, t) \frac{\sin \rho t}{\rho} d t+\int_{0}^{x} M(x-t) \frac{\sin \rho t}{\rho} d t \\
& +\int_{0}^{x} M(x-t)\left(\int_{0}^{t} K(t, \xi) \frac{\sin \rho \xi}{\rho} d \xi\right) d t=\rho \sin \rho x+\rho \int_{0}^{x} K(x, t) \sin \rho t d t .
\end{aligned}
$$

Integrating twice by parts the last integral we obtain

$$
\begin{aligned}
\int_{0}^{x}( & \left.-K_{x x}(x, t)+q(x) K(x, t)+M(x-t)+\int_{t}^{x} M(x-\xi) K(\xi, t) d \xi\right) \frac{\sin \rho t}{\rho} d t \\
& -K_{x}(x, t)_{\mid t=x} \frac{\sin \rho x}{\rho}-\frac{d K(x, x)}{d x} \frac{\sin \rho x}{\rho}-K(x, x) \cos \rho x+q(x) \frac{\sin \rho x}{\rho} \\
= & -K(x, x) \cos \rho x+K(x, 0)+K_{t}(x, t)_{\mid t=x} \frac{\sin \rho x}{\rho}-\int_{0}^{x} K_{t t}(x, t) \frac{\sin \rho t}{\rho} d t .
\end{aligned}
$$

Since

$$
K_{x}(x, t)_{\mid t=x}+K_{t}(x, t)_{\mid t=x}=\frac{d K(x, x)}{d x}, \quad K(x, 0)=0,
$$


it follows that

$$
\int_{0}^{x} A(x, t) \sin \rho t d t+B(x) \sin \rho x=0,
$$

where

$$
\begin{aligned}
A(x, t) & =K_{t t}(x, t)-K_{x x}(x, t)+q(x) K(x, t)+M(x-t)+\int_{t}^{x} M(x-\xi) K(\xi, t) d \xi, \\
B(x) & =q(x)-2 \frac{d K(x, x)}{d x} .
\end{aligned}
$$

Fix $x$ and take $\rho=\rho_{n}:=(2 \pi n+\pi / 2) x^{-1}$ in (10). Then

$$
\lim _{n \rightarrow \infty} \int_{0}^{x} A(x, t) \sin \left(\rho_{n} t x^{-1}\right) d t=0
$$

and consequently, (10) yields $B(x) \equiv 0$. Therefore, $\int_{0}^{x} A(x, t) \sin \rho t d t=0$ for all $\rho$, and hence $A(x, t)=0$ for $0 \leq t \leq x$. Lemma 1 is proved.

Lemma 2. Let $f \in C^{2}[0, \pi]$, and let

$$
h(x):=f(x)+\int_{0}^{x} K(x, t) f(t) d t .
$$

Then

$$
\ell_{1} h(x)=-f^{\prime \prime}(x)-\int_{0}^{x} K(x, t) f^{\prime \prime}(t) d t+f(0) K_{t}(x, t)_{\mid t=0}, x \in[0, \pi]
$$

Proof. Differentiating (11) twice we get

$$
\begin{aligned}
& h^{\prime}(x)=f^{\prime}(x)+K(x, x) f(x)+\int_{0}^{x} K_{x}(x, t) f(t) d t, \\
& h^{\prime \prime}(x)=f^{\prime \prime}(x)+\frac{d}{d x}(K(x, x) f(x))+K_{x}(x, t)_{\mid t=x} f(x)+\int_{0}^{x} K_{x x}(x, t) f(t) d t,
\end{aligned}
$$

and consequently,

$$
\begin{aligned}
\ell_{1} h(x)= & -f^{\prime \prime}(x)-\frac{d}{d x}(K(x, x) f(x))-K_{x}(x, t)_{\mid t=x} f(x)-\int_{0}^{x} K_{x x}(x, t) f(t) d t \\
& +q(x) f(x)+\int_{0}^{x} q(x) K(x, t) f(t) d t+\int_{0}^{x} M(x-t) f(t) d t \\
& +\int_{0}^{x} M(x-t)\left(\int_{0}^{t} K(t, \xi) f(\xi) d \xi\right) d t
\end{aligned}
$$

Taking (9) into account we infer

$$
\begin{aligned}
\ell_{1} h(x)= & -f^{\prime \prime}(x)+\int_{0}^{x} f(t)\left(-K_{x x}(x, t)+q(x) K(x, t)+M(x-t)+\int_{t}^{x} M(x-\xi) K(\xi, t) d \xi\right) d t \\
& -K_{x}(x, t)_{\mid t=x} f(x)-\frac{d K(x, x)}{d x} f(x)-K(x, x) f^{\prime}(x)+2 \frac{d K(x, x)}{d x} f(x) .
\end{aligned}
$$


Together with (8) this yields

$$
\ell_{1} h(x)=-f^{\prime \prime}(x)-\int_{0}^{x} f(t) K_{t t}(x, t) d t-K_{x}(x, t)_{\mid t=x} f(x)+\frac{d K(x, x)}{d x} f(x)-K(x, x) f^{\prime}(x) .
$$

Integrating the integral by parts twice we calculate

$$
\ell_{1} h(x)=-f^{\prime \prime}(x)-\int_{0}^{x} K(x, t) f^{\prime \prime}(t) d t+f(0) K_{t}(x, t)_{\mid t=0},
$$

i.e. (12) is valid. Lemma 2 is proved.

\section{The proof of Theorem 1}

For each fixed $t$, let $g(x, t, \lambda), x \geq t$ be the solution of the Cauchy problem

$$
\begin{aligned}
& -g^{\prime \prime}(x, t, \lambda)+q(x) g(x, t, \lambda)-\lambda g(x, t, \lambda)+\int_{t}^{x} M(x-\xi) g(\xi, t, \lambda) d \xi=0, \\
& g(t, t, \lambda)=0, \quad g_{x}(x, t, \lambda)_{\mid x=t}=1
\end{aligned}
$$

with respect to $x$. It is easy to check that

$$
u(x, \lambda)=\int_{0}^{x} g(x, t, \lambda) R(t) d t
$$

where $u(x, \lambda)$ was defined by (3). Fix $t$ in (13)-(14) and make the substitution $x=z+t$, $g_{1}(z, \lambda):=g(z+t, t, \lambda)$. Then (13)-(14) take the form

$$
\begin{gathered}
-g_{1}^{\prime \prime}(z, \lambda)+q(z+t) g_{1}(z, \lambda)-\lambda g_{1}(z, \lambda)+\int_{0}^{z} M(z-\tau) g_{1}(\tau, \lambda) d \tau=0, \\
g_{1}(0, \lambda)=0, \quad g_{1}^{\prime}(0, \lambda)=1 .
\end{gathered}
$$

Similar to (7) one gets the representation

$$
g_{1}(z, \lambda)=\frac{\sin \rho z}{\rho}+\int_{0}^{z} P_{1}(z, \tau, t) \frac{\sin \rho \tau}{\rho} d \tau,
$$

where $P_{1}(z, \tau, t)$ is a smooth function. Therefore,

$$
g(x, t, \lambda)=\frac{\sin \rho(x-t)}{\rho}+\int_{0}^{x-t} P(x, \tau, t) \frac{\sin \rho \tau}{\rho} d \tau,
$$

where $P(x, \tau, t)=P_{1}(z, \tau, t)$. Substituting (18) into (15) we get

$$
u(x, \lambda)=\int_{0}^{x}\left(\frac{\sin \rho(x-t)}{\rho}+\int_{0}^{x-t} P(x, \tau, t) \frac{\sin \rho \tau}{\rho} d \tau\right) R(t) d t,
$$

and consequently,

$$
u(x, \lambda)=\int_{0}^{x}\left(R(x-t)+\int_{0}^{x-t} P(x, t, \tau) R(\tau) d \tau\right) \frac{\sin \rho t}{\rho} d t .
$$


Substituting (19) into (4) we calculate

$$
\Delta(\lambda)=1-\int_{0}^{\pi} V(t) d t \int_{0}^{t}\left(R(t-s)+\int_{0}^{t-s} P(t, s, \tau) R(\tau) d \tau\right) \frac{\sin \rho s}{\rho} d s .
$$

This yields

$$
\Delta(\lambda)=1-\int_{0}^{\pi} \frac{\sin \rho s}{\rho}\left(\int_{s}^{\pi} V(t)\left(R(t-s)+\int_{0}^{t-s} P(t, s, \tau) R(\tau) d \tau\right) d t\right) d s
$$

or

$$
\Delta(\lambda)=1+\int_{0}^{\pi} B(t) \frac{\sin \rho t}{\rho} d t
$$

where

$$
B(t)=-\int_{t}^{\pi} V(s)\left(R(s-t)+\int_{0}^{s-t} P(s, t, \tau) R(\tau) d \tau\right) d s
$$

Changing the variable $s=\pi-\xi$ in (21) we infer

$$
B(t)=-\int_{0}^{\pi-t} V(\pi-\xi)\left(R(\pi-\xi-t)+\int_{0}^{\pi-\xi-t} P(\pi-\xi, t, \tau) R(\tau) d \tau\right) d \xi .
$$

Taking $x=\pi-t$, we rewrite the last relation in the following form

$$
B(\pi-x)=-\int_{0}^{x} V(\pi-\xi)\left(R(x-\xi)+\int_{0}^{x-\xi} P(\pi-\xi, \pi-x, \tau) R(\tau) d \tau\right) d \xi .
$$

It follows from (5) and (22) that $B(t) \in C[0, \pi]$, and

$$
B(\pi-x)=d x^{\alpha+\beta+1}(1+o(1)), x \rightarrow 0,
$$

where $d=-d_{0} d_{1} \neq 0$. Using (20) and (23) by the well-known method (see, for example, [10]) one gets that the function $\Delta(\lambda)$ is entire in $\lambda$ of order $1 / 2$, and

$$
\begin{aligned}
& \Delta(\lambda)=1+O\left(\rho^{-1}\right), \quad \lambda=\rho^{2}>0, \lambda \rightarrow+\infty, \\
&|\Delta(\lambda)| \geq C_{\delta}|\rho|^{-\gamma} \exp (|\operatorname{Im} \rho| \pi), \rho \in G_{\delta},
\end{aligned}
$$

where $\gamma=\alpha+\beta+3, G_{\delta}=\left\{\rho:\left|\rho-\rho_{n}\right| \geq \delta \forall n \geq 1\right\}, \lambda=\rho^{2}, \lambda_{n}=\rho_{n}^{2}, \delta>0$. According to (24) and Hadamard's factorization theorem, the function $\Delta(\lambda)$ is uniquely determined by its zeros. Under the assumptions of the theorem this yields

$$
\Delta(\lambda) \equiv \tilde{\Delta}(\lambda)
$$

We consider the function

$$
F(\lambda):=\frac{u(\pi, \lambda)-\tilde{u}(\pi, \lambda)}{\Delta(\lambda)} .
$$

Using (19), (25) and the assumptions of the theorem we conclude that the function $F(\lambda)$ is entire in $\lambda$, and $F(\lambda)=O\left(\lambda^{p}\right)$ as $|\lambda| \rightarrow \infty$, with $p \geq 0$. By Liouville's theorem one gets that $F(\lambda)$ 
is a polynomial. On the other hand, taking (19) and (24) into account we infer that $F(\lambda)$ tends to zero as $\lambda>0, \lambda \rightarrow+\infty$. Therefore $F(\lambda) \equiv 0$, i.e.

$$
u(\pi, \lambda) \equiv \tilde{u}(\pi, \lambda)
$$

Furthermore, it follows from (19) and (27) that

$$
\int_{0}^{\pi}\left(R(\pi-t)-\tilde{R}(\pi-t)+\int_{0}^{\pi-t} P(\pi, t, \tau)(R(\tau)-\tilde{R}(\tau)) d \tau\right) \sin \rho t d t \equiv 0,
$$

and consequently,

$$
R(\pi-t)-\tilde{R}(\pi-t)+\int_{0}^{\pi-t} P(\pi, t, \tau)(R(\tau)-\tilde{R}(\tau)) d \tau \equiv 0,
$$

or

$$
R(x)-\tilde{R}(x)+\int_{0}^{x} P(\pi, \pi-x, \tau)(R(\tau)-\tilde{R}(\tau)) d \tau \equiv 0 .
$$

Since this homogeneous integral equation has only the trivial solution it follows that

$$
R(x)=\tilde{R}(x), \quad x \in[0, \pi] .
$$

Let the functions $u_{0}(x, \lambda)$ and $R_{0}(x)$ be the solutions of the integral equations

$$
u(x, \lambda)=u_{0}(x, \lambda)+\int_{0}^{x} K(x, t) u_{0}(t, \lambda) d t, R(x)=R_{0}(x)+\int_{0}^{x} K(x, t) R_{0}(t) d t .
$$

In view of (3) and (29),

$$
u_{0}(0, \lambda)=u_{0}^{\prime}(0, \lambda)=0, \ell_{1} u(x, \lambda)=\lambda u(x, \lambda)-R(x) .
$$

Moreover, applying Lemma 2 one gets

$$
\ell_{1} u(x, \lambda)=-u_{0}^{\prime \prime}(x, \lambda)-\int_{0}^{x} K(x, t) u_{0}^{\prime \prime}(t, \lambda) d t .
$$

It follows from (29) and () that

$$
\ell_{1} u(x, \lambda)=\lambda u_{0}(x, \lambda)+\lambda \int_{0}^{x} K(x, t) u_{0}(t, \lambda) d t-R_{0}(x)-\int_{0}^{x} K(x, t) R_{0}(t) d t .
$$

Comparing this relation with (30) we obtain

$$
u_{0}^{\prime \prime}(x, \lambda)+\lambda u_{0}(x, \lambda)-R_{0}(x)+\int_{0}^{x} K(x, t)\left(u_{0}^{\prime \prime}(t, \lambda)+\lambda u_{0}(t, \lambda)-R_{0}(t)\right) d t=0,
$$

and consequently,

$$
u_{0}^{\prime \prime}(x, \lambda)+\lambda u_{0}(x, \lambda)=R_{0}(x)
$$


The solution of the Cauchy problem (31), () has the form

$$
u_{0}(x, \lambda)=\int_{0}^{x} \frac{\sin \rho(x-t)}{\rho} R_{0}(t) d t .
$$

Denote

$$
V_{0}(x)=V(x)+\int_{x}^{\pi} K(t, x) V(t) d t .
$$

It follows from (4), (29) and (33) that

$$
\Delta(\lambda)=1-\int_{0}^{\pi} V_{0}(t) u_{0}(t, \lambda) d t .
$$

Taking (28), (29) and (32) into account we obtain $u_{0}(x, \lambda) \equiv \tilde{u}_{0}(x, \lambda)$. Together with (26) and (34) this yields

$$
\int_{0}^{\pi}\left(V_{0}(t)-\tilde{V}_{0}(t)\right) u_{0}(t, \lambda) d t \equiv 0
$$

Using (32) again we calculate

$$
\int_{0}^{\pi}\left(\int_{s}^{\pi}\left(V_{0}(t)-\tilde{V}_{0}(t)\right) R_{0}(t-s) d t\right) \sin \rho s d s \equiv 0,
$$

and consequently,

$$
\int_{s}^{\pi}\left(V_{0}(t)-\tilde{V}_{0}(t)\right) R_{0}(t-s) d t \equiv 0, s \in[0, \pi]
$$

or

$$
\int_{0}^{x}\left(V_{0}(\pi-\xi)-\tilde{V}_{0}(\pi-\xi)\right) R_{0}(x-\xi) d \xi \equiv 0, x \in[0, \pi] .
$$

Applying Titchmarsh's theorem we infer $V_{0}(\pi-\xi) \equiv \tilde{V}_{0}(\pi-\xi), \xi \in[0, \pi]$ or

$$
V_{0}(x) \equiv \tilde{V}_{0}(x), x \in[0, \pi]
$$

By virtue of (33) this yields

$$
V(x) \equiv \tilde{V}(x), x \in[0, \pi]
$$

and Theorem 1 is proved.

\section{Acknowledgement}

This research was supported in part by Grants 10-01-00099 and 10-01-92001-NSC of Russian Foundation for Basic Research and Taiwan National Science Council. 


\section{References}

[1] V. A. Marchenko, Sturm-Liouville operators and their applications. "Naukova Dumka", Kiev, 1977; English transl., Birkhäuser, 1986.

[2] B. M. Levitan, Inverse Sturm-Liouville problems. Nauka, Moscow, 1984; English transl., VNU Sci.Press, Utrecht, 1987.

[3] G. Freiling and V. A. Yurko, Inverse Sturm-Liouville Problems and their Applications. NOVA Science Publishers, New York, 2001.

[4] R. Beals, P. Deift and C. Tomei, Direct and inverse scattering on the line. Mathematica Surveys and Monographs, 28. AMS, Providence, RI, 1988.

[5] V. A. Yurko, Method of Spectral Mappings in the Inverse Problem Theory, Inverse and Ill-posed Problems Series. VSP, Utrecht, 2002, 303pp.

[6] V. A. Yurko, An inverse problem for integro-differential operators. Mat. zametki, 50, (1991), $134-146$ (Russian); English transl. in Math. Notes, 50, no.5-6 (1991), 1188-1197.

[7] V. A. Yurko, An inverse problem for integral operators, Mat. Zametki, 37, (1985), 690-701 (Russian); English transl. in Math.Notes, 37, (1985), 378-385.

[8] Yu. Kuryshova, The inverse spectral problem for integro-differential operators, Mat. Zametki, 81 (2007), 855866; English translation in Math. Notes, 81, (2007), 767-777.

[9] S. Buterin, On an inverse spectral problem for a convolution integro- differential operator, Results in Mathematics, 50 (2007), 173-181.

[10] Naimark M.A., Linear Differential Operators, 2nd ed., Nauka, Moscow, 1969; English transl. of 1st ed., Parts I,II, Ungar, New York, 1967, 1968.

Department of Mathematics, Saratov State University, Astrakhanskaya 83, Saratov 410012, Russia.

E-mail: jvkuryshova@rambler.ru 